\title{
The relationship between HPV and penile cancer: Filling a knowledge gap in the general population
}

\author{
Julio Slongo, MD'; Anna R. Giuliano, MD'; Peter A. Johnstone, MD³, Philippe E. Spiess, $M D^{1}$ \\ 'Department of Genitourinary Oncology, Moffitt Cancer Center, Tampa, FL, United States; ${ }^{2}$ Center of Infection Research in Cancer, Moffiitt Cancer Center, Tampa, FL, United States; ${ }^{3}$ Department of Radiation \\ Oncology, Moffitt Cancer Center, Tampa, FL, United States
}

Cite as: Can Urol Assoc J 2019;13(2):38. http://dx.doi.org/10.5489/cuaj.5838

See related article on page 32

$\mathrm{T}$ he interesting study by Zavaski et al highlights the significant need for dissemination of accurate and actionable human papillomavirus (HPV) and cancer education aimed at the general population. ${ }^{1}$ The analysis of the Health Information National Trends Survey (HINTS) reveals concerning facts about how little the sampled population of Americans knows about HPV and penile cancer; in this study, less than $65 \%$ of the study cohort had heard any information about HPV, with less than $30 \%$ being aware that HPV causes penile cancer. Considering the characteristics of the study, these results are probably an optimistic estimate of the proportion of Americans that have knowledge of HPV and the cancers these infections cause. The majority of participants who completed this questionnaire were young, highly educated ( $40 \%$ with college degree), had a higher income, and lived in urban areas; characteristics in sharp contrast to those of the typical high-risk penile cancer population. Therefore, we can assume that the true risk population for penile cancer has little to no knowledge about this important association, providing an impetus to broaden education and potential prevention campaigns to include use of social media to reach and educate the younger patient population.

When knowledge about HPV and its association to penile cancer was compared with knowledge to its association to cervical cancer, which has been shown to be as high as $80 \%$ in some observational studies, this again demonstrates that urologists and healthcare providers in general have a critical task to provide risk-based and appropriate patient education.

An important lesson should be taken from our gynecology colleagues in regard to patient education and cancer prevention: based on multiple strategies, ranging from detailed in-office conversations and education of primary care providers to media information dissemination, they were able to increase consciousness of the risk of HPV and cervical cancer. The gynecological literature has a plethora of examples that show how patient-targeted education can be successful.
This issue becomes even more problematic when we realize that education would decrease the incidence of penile cancer if we are able to increase the number of boys vaccinated with the HPV vaccine. The association of HPV, cervical cancer and HPV vaccine protection is highly disseminated in the general population, but little to no information is disseminated regarding prevention of penile cancer. Obviously, the incidence of those two malignancies are very different, but the protection benefit that can be obtained from vaccination in both cases should be voiced in an unequivocal manner to the general public. Once again, with increased education about the benefit of vaccinating boys, particularly targeting pediatric and primary healthcare providers in outpatient and acute care clinics, the number of vaccinations would likely significantly increase.

The interesting results presented by Zavaski et al unquestionably highlight the necessity for consistent and routine education by all health providers caring for at-risk men and women. Penile cancer therapeutic outcomes have not improved significantly over the past three decades, with uniformly poor prognoses for advanced stages. Therefore, prevention of this disease, as well as early diagnosis and treatment is essential to improve outcomes. To achieve this, patient education is fundamental.

We congratulate the authors in their effort to rigorously demonstrate the current lack of knowledge on this important subject and imploring the medical community to embrace it as an opportunity for cancer prevention in a young patient population.

Competing interests: The authors report no competing personal or financial interest related to this work.

\section{Reference}

1. Zavaski ME, Hanske J, Löppenberg B, et al. Contemporary perceptions of human papillomavirus and penile cancer: Perspectives from a national survey. Can Urol Assoc J 2018 July 31; Epub ahead of print. http://dx.doi.org/10.5489/cuai.5366

Correspondence: Dr. Julio Slongo, Department of Genitourinary Oncology, Moffitt Cancer Center, Tampa, FL, United States; julioslongo@health.usf.edu 